\title{
The fractional modified Zakharov system for plasmas with a quantum correction
}

\author{
Lang $\mathrm{Li}^{\dagger}$, Lingyu Jin ${ }^{\dagger}$, Changping $\mathrm{Xie}^{\dagger}$ and Shaomei Fang ${ }^{*}$
}

${ }^{\text {*Correspondence: }}$ dz90@scau.edu.cn

Department of Applied Mathematics, South China Agricultural University, Wushan Road, Guangzhou, 510642, China

†Equal contributors
$2015 \mathrm{Li}$ et al. This article is distributed under the terms of the Creative Commons Attribution 4.0 International License (http://creativecommons.org/licenses/by/4.0/), which permits unrestricted use, distribution, and reproduction in any medium, provided you give appropriate credit to the original author(s) and the source, provide a link to the Creative Commons license, and indicate if changes were made.

\section{Introduction}

In this paper, we consider the following fractional modified Zakharov system

$$
\begin{aligned}
& i \partial_{t} E+\partial_{x x} E-H^{2}\left(-\partial_{x x}\right)^{\alpha} E=n E, \\
& \partial_{t t} n-\partial_{x x} n+H^{2}\left(-\partial_{x x}\right)^{\beta} n=\partial_{x x}\left(|E|^{2}\right), \\
& E(x, 0)=E_{0}(x), \quad n(x, 0)=n_{0}(x), \quad \partial_{t} n(x, 0)=n_{1}(x), \\
& E(x+2 \pi, t)=E(x, t), \quad n(x+2 \pi, t)=n(x, t), \quad \partial_{t} n(x+2 \pi, t)=\partial_{t} n(x, t),
\end{aligned}
$$

where $1<\alpha \leq \beta<2, E: \mathbb{R}^{1+1} \rightarrow \mathbb{C}$ represents the slowly varying envelope of the highfrequency electric field, $n: \mathbb{R}^{1+1} \rightarrow \mathbb{R}$ denotes the low-frequency variation of the density of the ions and $H$ is the dimensionless quantum parameter given by the ratio of the ion plasmon and electron thermal energies.

When $H=0$, (1)-(2) reduce to the classical Zakharov system. It is well known that the classical Zakharov system [1], which describes the nonlinear interaction of Langmuir waves and ion-acoustic waves, is one of the most important models in plasma physics. Physically, many authors have paid much attention to investigate the nonlinear properties of this system, such as the existence of solitons, chaos, hyperchaos, Hopf bifurcation. For more details, see [2-4]. Mathematically, this system was also broadly studied concerning its existence, uniqueness and regularity of the solutions, see [5-8].

When $H>0, \alpha=2, \beta=2$, it was exactly the Garcia model [9] which obtained by using a quantum fluid approach. Since this system took into account the quantum corrections, it has became more important on the investigation of the quantum counterpart of some of the plasma physics phenomena $[10,11]$. In this sense, (1)-(2) can be regarded as a fractional generalization of the Garcia model. 
The interest in the study of differential equations of fractional order lies in the fact that fractional derivatives provide a powerful and useful tool for the description of memory and hereditary properties of various material and process. With this advantage, particularly in some interdisciplinary fields, the fractional order models become more realistic and practical than the classical integer-order models [12]. Recently, the fractional differential equations have been attracting great attention and widely been used in the areas of physics, engineering, chemistry, biology, economics, control theory, signal and image processing, biophysics, aerodynamics, etc. [13-15]. For example, the authors [16] studied the chaotic behavior of a generalization of the Liu system with fractional order. In [17], the existence and uniqueness of the solution for fractional neutral differential equations with infinite delay was obtained. Other new results concerning the numerical investigation on the dynamics and properties of the system for fractional differential equations can be found in [18-25], etc.

Concerning the mathematical issues for (1)-(2), You et al. [25] established the global existence of the solution to (1)-(2) with initial boundary conditions via a priori estimates and Galerkin method when $H>0, \alpha=2, \beta=2$. Jin et al. obtained the existence of weak solutions and the global attractor for modified Zakharov equations with a quantum correction $[26,27]$. To our knowledge, there is no general existence theory that has been developed for (1)-(4).

In this paper, we are interested in studying the global existence and uniqueness of the weak solution. Motivated by [24], we combine the energy method with a priori estimates to establish the existence of the weak solution in some fractional Sobolev spaces. To overcome the mathematical difficulty arises in the fractional model, we use the properties of fractional Sobolev spaces and the linear interpolation to deal with the nonlinear terms.

This paper is organized as follows. In Section 2, we obtain a priori estimates. In Section 3, we prove the existence and uniqueness of global solution to the problem (1)-(4). In Section 4, we give a conclusion for our results.

Now we give some notations.

Let $\Omega=[0,2 \pi]$. We denote by $L^{p}(\Omega)$ the space of all the $p$ th integrable functions $f$ normed by

$$
\|f\|_{L^{p}(\Omega)}=\left(\int_{\Omega}|f(x)|^{p} d x\right)^{\frac{1}{p}}, \quad\|f\|_{L^{\infty}(\Omega)}=\underset{\Omega}{\operatorname{ess} \sup }|f(x)| .
$$

When no confusion arises, we set $L^{p}:=L^{p}(\Omega)$ for $1 \leq p \leq \infty$.

The space $L^{p}(0, T ; X)$ consists of all measurable functions $f:[0, T] \rightarrow X$ with

$$
\|f\|_{L^{p}(0, T ; X)}=\left(\int_{0}^{T}\|f\|_{X}^{p} d t\right)^{\frac{1}{p}}<\infty
$$

for $1 \leq p<\infty$, and

$$
\|f\|_{L^{\infty}(0, T ; X)}=\underset{0 \leq t \leq T}{\operatorname{ess} \sup }\|f\|_{X}<\infty .
$$

If $u$ is a periodic function, we can express it by Fourier series and write

$$
u=\sum_{j \in \mathbb{Z}} a_{j} e^{i j \cdot x} .
$$


$\Lambda^{\alpha} u$ can be defined by

$$
\Lambda^{\alpha} u=\sum_{j \in \mathbb{Z}}|j|^{\alpha} a_{j} e^{i j \cdot x}
$$

where $\Lambda:=(-\Delta)^{\frac{1}{2}}$ is the so-called Zygmund operator.

Define

$$
A=\left\{\left.u\left|u=\sum_{j \in \mathbb{Z}} a_{j} e^{i j \cdot x}, \sum_{j \in \mathbb{Z}} a_{j}^{2}<\infty, \sum_{j \in \mathbb{Z}}\right| j\right|^{2 \alpha} a_{j}^{2}<\infty\right\} .
$$

$H^{\alpha}$ is a complete space of $A$ with the induced norm

$$
\|u\|_{H^{\alpha}}=\left(\sum_{j \in \mathbb{Z}} a_{j}^{2}\right)^{\frac{1}{2}}+\left(\sum_{j \in \mathbb{Z}}|j|^{2 \alpha} a_{j}^{2}\right)^{\frac{1}{2}} .
$$

Then $H^{\alpha}$ is a Banach space. It is easy to show that $H^{\alpha}$ is a Hilbert space with the inner product

$$
(u, v)_{H^{\alpha}}=\left(\Lambda^{\alpha} u, \Lambda^{\alpha} v\right)=\sum_{j \in \mathbb{Z}}|j|^{2 \alpha} a_{j} b_{j}
$$

where $\Lambda^{\alpha} v=\sum_{j \in \mathbb{Z}}|j|^{\alpha} b_{j} e^{i j \cdot x}$.

We denote by $C$ a positive constant which may change from one line to the next line.

\section{A priori estimates}

To study the solution of the fractional system, we bring in $\phi$, and transform (1)-(4) into the following form

$$
\begin{aligned}
& i \partial_{t} E+\partial_{x x} E-H^{2} \Lambda^{2 \alpha} E=n E, \\
& \partial_{t} n-\partial_{x x} \phi=0, \\
& \partial_{t} \phi-n-H^{2} \Lambda^{2(\beta-1)} n-|E|^{2}=0, \\
& E(x, 0)=E_{0}(x), \quad n(x, 0)=n_{0}(x), \quad \phi(x, 0)=\phi_{0}(x), \\
& E(x+2 \pi, t)=E(x, t), \quad n(x+2 \pi, t)=n(x, t), \quad \phi(x+2 \pi, t)=\phi(x, t) .
\end{aligned}
$$

Lemma 1 Assume that $E_{0}(x) \in L^{2}(\Omega)$, then we have

$$
\|E(x, t)\|_{L^{2}}^{2}=\left\|E_{0}(x)\right\|_{L^{2}}^{2} .
$$

Proof Multiplying (5) by $\bar{E}$, integrating over $\Omega$, and then taking the imaginary part, we have

$$
\frac{1}{2} \frac{d}{d t}\|E\|_{L^{2}}^{2}=0
$$

This completes the proof. 
Lemma 2 Assume that $E_{0}(x) \in H^{\alpha}(\Omega), n_{0}(x) \in H^{\beta-1}(\Omega), \phi_{0}(x) \in H^{1}(\Omega)$, then we have

$$
\sup _{0 \leq t \leq T}\left(\left\|\partial_{x} E\right\|_{L^{2}}^{2}+\left\|\partial_{x} \phi\right\|_{L^{2}}^{2}+\left\|\Lambda^{\alpha} E\right\|_{L^{2}}^{2}+\|n\|_{L^{2}}^{2}+\left\|\Lambda^{\beta-1} n\right\|_{L^{2}}^{2}\right) \leq C,
$$

where $C$ is a positive constant depending on $\left\|E_{0}\right\|_{H^{\alpha}},\left\|n_{0}\right\|_{H^{\beta-1}},\left\|\phi_{0}\right\|_{H^{1}}$, and $T$.

Proof Multiplying (6) by $n$, and integrating over $\Omega$, we have

$$
\frac{1}{2} \frac{d}{d t}\|n\|_{L^{2}}^{2}-\int_{\Omega} \partial_{x x} \phi n d x=0
$$

Since

$$
\begin{aligned}
\int_{\Omega} \partial_{x x} \phi n d x & =\int_{\Omega} \partial_{x x} \phi\left(\partial_{t} \phi-H^{2} \Lambda^{2(\beta-1)} n-|E|^{2}\right) d x \\
& =-\frac{1}{2} \frac{d}{d t}\left\|\partial_{x} \phi\right\|_{L^{2}}^{2}-\frac{H^{2}}{2} \frac{d}{d t}\left\|\Lambda^{(\beta-1)} n\right\|_{L^{2}}^{2}-\int_{\Omega} \partial_{t} n|E|^{2} d x
\end{aligned}
$$

it follows that

$$
\frac{1}{2} \frac{d}{d t}\left(\|n\|_{L^{2}}^{2}+\left\|\partial_{x} \phi\right\|_{L^{2}}^{2}+H^{2}\left\|\Lambda^{(\beta-1)} n\right\|_{L^{2}}^{2}\right)+\int_{\Omega} \partial_{t} n|E|^{2} d x=0 .
$$

Multiplying (5) by $\overline{\partial_{t} E}$, integrating over $\Omega$, and taking the real part, we have

$$
\frac{1}{2} \frac{d}{d t}\left\|\partial_{x} E\right\|_{L^{2}}^{2}+\frac{H^{2}}{2} \frac{d}{d t}\left\|\Lambda^{\alpha} E\right\|_{L^{2}}^{2}+\frac{1}{2} \int_{\Omega} n \partial_{t}\left(|E|^{2}\right) d x=0 .
$$

Since

$$
\frac{d}{d t} \int_{\Omega} n|E|^{2} d x=\int_{\Omega} \partial_{t} n|E|^{2} d x+\int_{\Omega} n \partial_{t}\left(|E|^{2}\right) d x
$$

from (10) and (11), we can deduce that

$$
\begin{aligned}
& \frac{d}{d t}\left(\frac{1}{2}\|n\|_{L^{2}}^{2}+\frac{1}{2}\left\|\partial_{x} \phi\right\|_{L^{2}}^{2}+\frac{H^{2}}{2}\left\|\Lambda^{(\beta-1)} n\right\|_{L^{2}}^{2}+\left\|\partial_{x} E\right\|_{L^{2}}^{2}\right. \\
& \left.\quad+H^{2}\left\|\Lambda^{\alpha} E\right\|_{L^{2}}^{2}+\int_{\Omega} n\left(|E|^{2}\right) d x\right)=0 .
\end{aligned}
$$

Set

$$
\begin{aligned}
\Psi(t)= & \frac{1}{2}\|n\|_{L^{2}}^{2}+\frac{1}{2}\left\|\partial_{x} \phi\right\|_{L^{2}}^{2}+\frac{H^{2}}{2}\left\|\Lambda^{(\beta-1)} n\right\|_{L^{2}}^{2}+\left\|\partial_{x} E\right\|_{L^{2}}^{2} \\
& +H^{2}\left\|\Lambda^{\alpha} E\right\|_{L^{2}}^{2}+\int_{\Omega} n\left(|E|^{2}\right) d x
\end{aligned}
$$

we have

$$
\Psi(t)=\Psi(0)
$$


By using the Hölder inequality, the Gagliardo-Nirenberg inequality, and Lemma 1, we have

$$
\begin{aligned}
& \frac{1}{2}\|n\|_{L^{2}}^{2}+\frac{1}{2}\left\|\partial_{x} \phi\right\|_{L^{2}}^{2}+\frac{H^{2}}{2}\left\|\Lambda^{(\beta-1)} n\right\|_{L^{2}}^{2}+\left\|\partial_{x} E\right\|_{L^{2}}^{2}+H^{2}\left\|\Lambda^{\alpha} E\right\|_{L^{2}}^{2} \\
& \quad \leq \Psi(0)+\left.\left|\int_{\Omega} n\right| E\right|^{2} d x \mid \\
& \quad \leq \Psi(0)+\frac{1}{4}\|n\|_{L^{2}}^{2}+\|E\|_{L^{4}}^{4} \\
& \quad \leq \Psi(0)+\frac{1}{4}\|n\|_{L^{2}}^{2}+C\|E\|_{L^{2}}^{3}\left\|\partial_{x} E\right\|_{L^{2}} \\
& \quad \leq \Psi(0)+\frac{1}{4}\|n\|_{L^{2}}^{2}+\frac{1}{4}\left\|\partial_{x} E\right\|_{L^{2}}^{2}+C .
\end{aligned}
$$

Owing to the inequality

$$
\begin{aligned}
\Psi(0) & \leq C\left(\left\|n_{0}\right\|_{L^{2}}^{2}+\left\|\partial_{x} \phi_{0}\right\|_{L^{2}}^{2}+\left\|\Lambda^{(\beta-1)} n_{0}\right\|_{L^{2}}^{2}+\left\|\partial_{x} E_{0}\right\|_{L^{2}}^{2}+\left\|\Lambda^{\alpha} E_{0}\right\|_{L^{2}}^{2}\right) \\
& \leq C\left(\left\|E_{0}\right\|_{H^{\alpha}}+\left\|n_{0}\right\|_{H^{\beta-1}}+\left\|\phi_{0}\right\|_{H^{1}}^{2}\right)
\end{aligned}
$$

we obtain the desired result.

Lemma 3 Assume that $E_{0}(x) \in H^{2 \alpha}(\Omega), n_{0}(x) \in H^{\beta}(\Omega), \phi_{0}(x) \in H^{2}(\Omega)$, then we have

$$
\sup _{0 \leq t \leq T}\left(\left\|\partial_{t} E\right\|_{L^{2}}^{2}+\left\|\partial_{t} n\right\|_{L^{2}}^{2}+\left\|\partial_{t} \phi\right\|_{L^{2}}^{2}+\left\|\Lambda^{2 \alpha} E\right\|_{L^{2}}^{2}+\left\|\Lambda^{\beta} n\right\|_{L^{2}}^{2}+\left\|\partial_{x x} \phi\right\|_{L^{2}}^{2}\right) \leq C
$$

where $C$ is a positive constant depending on $\left\|E_{0}\right\|_{H^{2 \alpha}},\left\|n_{0}\right\|_{H^{\beta}},\left\|\phi_{0}\right\|_{H^{2}}$ and $T$.

Proof Differentiating (5) with respect to t, then multiplying it by $\bar{E}_{t}$, integrating over $\Omega$, and taking the imaginary part, we have

$$
\frac{1}{2} \frac{d}{d t}\left\|\partial_{t} E\right\|_{L^{2}}^{2}=\operatorname{Im} \int_{\Omega} \partial_{t}(n E) \bar{E}_{t} d x
$$

Therefore

$$
\begin{aligned}
\frac{d}{d t}\left\|\partial_{t} E\right\|_{L^{2}}^{2} & =2 \operatorname{Im} \int_{\Omega} \partial_{t}(n E) \bar{E}_{t} d x \\
& =2 \operatorname{Im} \int_{\Omega} \partial_{t} n E \bar{E}_{t} d x \\
& \leq 2\|E\|_{L^{\infty}}\left\|\partial_{t} n\right\|_{L^{2}}\left\|\partial_{t} E\right\|_{L^{2}} \\
& \leq C\left(\left\|\partial_{t} n\right\|_{L^{2}}^{2}+\left\|\partial_{t} E\right\|_{L^{2}}^{2}\right)
\end{aligned}
$$

where we have used the fact $\|E\|_{L^{\infty}} \leq C\left\|\partial_{x} E\right\|_{L^{2}}$ and Lemma 2 .

Differentiating (6) with respect to $t$, then multiplying it by $\partial_{t} n$, and integrating over $\Omega$, we have

$$
\frac{1}{2} \frac{d}{d t}\left\|\partial_{t} n\right\|_{L^{2}}^{2}+\frac{1}{2} \frac{d}{d t}\left\|\partial_{x} n\right\|_{L^{2}}^{2}+\frac{H^{2}}{2} \frac{d}{d t}\left\|\Lambda^{\beta} n\right\|_{L^{2}}^{2}=\int_{\Omega} \partial_{t} n \partial_{x x}\left(|E|^{2}\right) d x
$$


By using the Hölder inequality, the Gagliardo-Nirenberg inequality, and Lemma 2, we have

$$
\begin{aligned}
\int_{\Omega} \partial_{t} n \partial_{x x}\left(|E|^{2}\right) d x & \leq C\left(\left\|\partial_{t} n\right\|_{L^{2}}^{2}+\left\|\partial_{x x}\left(|E|^{2}\right)\right\|_{L^{2}}^{2}\right) \\
& \leq C\left(\left\|\partial_{t} n\right\|_{L^{2}}^{2}+\|E\|_{L^{\infty}}^{2}\left\|\partial_{x x} E\right\|_{L^{2}}^{2}+\left\|\partial_{x} E\right\|_{L^{4}}^{4}\right) \\
& \leq C\left(\left\|\partial_{t} n\right\|_{L^{2}}^{2}+\left\|\partial_{x x} E\right\|_{L^{2}}^{2}+1\right) \\
& \leq C\left(\left\|\partial_{t} n\right\|_{L^{2}}^{2}+\left\|\Lambda^{2 \alpha} E\right\|_{L^{2}}^{2}+1\right) .
\end{aligned}
$$

From (5), it is easy to see that

$$
\left\|\Lambda^{2 \alpha} E\right\|_{L^{2}}^{2} \leq C\left(\left\|\partial_{t} E\right\|_{L^{2}}^{2}+\left\|\partial_{x x} E\right\|_{L^{2}}^{2}+\|n E\|_{L^{2}}^{2}\right)
$$

By using the Gagliardo-Nirenberg inequality, the $\epsilon$-Young inequality and Lemma 2, we have

$$
\begin{aligned}
\left\|\Lambda^{2 \alpha} E\right\|_{L^{2}}^{2} & \leq C\left(\left\|\partial_{t} E\right\|_{L^{2}}^{2}+\left\|\Lambda^{2 \alpha} E\right\|_{L^{2}}^{\frac{2}{\alpha}}\|E\|_{L^{2}}^{2-\frac{2}{\alpha}}+\|E\|_{L^{\infty}}^{2}\|n\|_{L^{2}}^{2}\right) \\
& \leq \frac{1}{2}\left\|\Lambda^{2 \alpha} E\right\|_{L^{2}}^{2}+C\left(\left\|\partial_{t} E\right\|_{L^{2}}^{2}+1\right) .
\end{aligned}
$$

Thus

$$
\left\|\Lambda^{2 \alpha} E\right\|_{L^{2}}^{2} \leq C\left(\left\|\partial_{t} E\right\|_{L^{2}}^{2}+1\right) .
$$

Consequently

$$
\begin{aligned}
& \frac{1}{2} \frac{d}{d t}\left\|\partial_{t} n\right\|_{L^{2}}^{2}+\frac{1}{2} \frac{d}{d t}\left\|\partial_{x} n\right\|_{L^{2}}^{2}+\frac{H^{2}}{2} \frac{d}{d t}\left\|\Lambda^{\beta} n\right\|_{L^{2}}^{2} \\
& \quad \leq C\left(\left\|\partial_{t} n\right\|_{L^{2}}^{2}+\left\|\partial_{t} E\right\|_{L^{2}}^{2}+1\right) .
\end{aligned}
$$

From (12) and (14), we have

$$
\begin{aligned}
& \frac{d}{d t}\left(\left\|\partial_{t} E\right\|_{L^{2}}^{2}+\left\|\partial_{t} n\right\|_{L^{2}}^{2}+\left\|\partial_{x} n\right\|_{L^{2}}^{2}+H^{2}\left\|\Lambda^{\beta} n\right\|_{L^{2}}^{2}\right) \\
& \quad \leq C\left(\left\|\partial_{t} n\right\|_{L^{2}}^{2}+\left\|\partial_{t} E\right\|_{L^{2}}^{2}+1\right)
\end{aligned}
$$

Thus the Gronwall inequality yields the estimate

$$
\sup _{0 \leq t \leq T}\left(\left\|\partial_{t} E\right\|_{L^{2}}^{2}+\left\|\partial_{t} n\right\|_{L^{2}}^{2}+\left\|\partial_{x} n\right\|_{L^{2}}^{2}+\left\|\Lambda^{\beta} n\right\|_{L^{2}}^{2}\right) \leq C,
$$

where $C$ is a positive constant depending on $\left\|E_{0}\right\|_{H^{2 \alpha}},\left\|n_{0}\right\|_{H^{\beta}},\left\|\phi_{0}\right\|_{H^{2}}$, and $T$.

Then (6) and (13) imply

$$
\left\|\partial_{x x} \phi\right\|_{L^{2}}^{2}+\left\|\Lambda^{2 \alpha} E\right\|_{L^{2}}^{2} \leq C .
$$

From (7), we can also obtain

$$
\left\|\partial_{t} \phi\right\|_{L^{2}}^{2} \leq C
$$

where we have used the fact $\beta-1<\frac{\beta}{2}$. Thus we complete the proof. 
Lemma 4 Assume that $E_{0}(x) \in H^{4 \alpha}(\Omega), n_{0}(x) \in H^{2 \beta}(\Omega), \phi_{0}(x) \in H^{\beta+2}(\Omega)$, then we have

$$
\sup _{0 \leq t \leq T}\left(\left\|\partial_{t t} E\right\|_{L^{2}}^{2}+\left\|\partial_{t t} n\right\|_{L^{2}}^{2}+\left\|\partial_{t t} \phi\right\|_{L^{2}}^{2}+\left\|\Lambda^{2 \alpha} \partial_{t} E\right\|_{L^{2}}^{2}+\left\|\Lambda^{\beta} \partial_{t} n\right\|_{L^{2}}^{2}+\left\|\partial_{x x} \partial_{t} \phi\right\|_{L^{2}}^{2}\right) \leq C
$$

where $C$ is a positive constant depending on $\left\|E_{0}\right\|_{H^{4 \alpha}},\left\|n_{0}\right\|_{H^{2 \beta}},\left\|\phi_{0}\right\|_{H^{\beta+2}}$, and $T$.

Proof Differentiating (6) with respect to $t$ twice, multiplying it by $\partial_{t t} n$, and integrating over $\Omega$, we have

$$
\frac{d}{d t}\left(\left\|\partial_{t t} n\right\|_{L^{2}}^{2}+\left\|\partial_{x} \partial_{t} n\right\|_{L^{2}}^{2}+H^{2}\left\|\Lambda^{\beta} \partial_{t} n\right\|_{L^{2}}^{2}\right)-2 \int_{\Omega} \partial_{t} \partial_{x x}\left(|E|^{2}\right) \partial_{t t} n d x=0
$$

By using the Hölder inequality, the Gagliardo-Nirenberg inequality, and Lemma 3, we have

$$
\begin{aligned}
& \left|\int_{\Omega} \partial_{t} \partial_{x x}\left(|E|^{2}\right) \partial_{t t} n d x\right| \\
& \quad \leq C\left(\left\|\partial_{t t} n\right\|_{L^{2}}^{2}+\left\|\partial_{t} \partial_{x x}\left(|E|^{2}\right)\right\|_{L^{2}}^{2}\right) \\
& \quad \leq C\left(\left\|\partial_{t t} n\right\|_{L^{2}}^{2}+\left\|\partial_{t} \partial_{x x} E\right\|_{L^{2}}^{2}\|E\|_{L^{\infty}}^{2}+\left\|\partial_{t} \partial_{x} E\right\|_{L^{2}}^{2}\left\|\partial_{x} E\right\|_{L^{\infty}}^{2}\right) \\
& \quad \leq C\left(\left\|\partial_{t t} n\right\|_{L^{2}}^{2}+\left\|\Lambda^{2 \alpha} \partial_{t} E\right\|_{L^{2}}^{2}+1\right) .
\end{aligned}
$$

Since

$$
i \partial_{t t} E+\partial_{x x} \partial_{t} E-H^{2} \Lambda^{2 \alpha} \partial_{t} E-\partial_{t}(n E)=0,
$$

we have

$$
\begin{aligned}
\left\|\Lambda^{2 \alpha} \partial_{t} E\right\|_{L^{2}}^{2} & \leq C\left(\left\|\partial_{t t} E\right\|_{L^{2}}^{2}+\left\|\partial_{x x} \partial_{t} E\right\|_{L^{2}}^{2}+\left\|\partial_{t}(n E)\right\|_{L^{2}}^{2}\right) \\
& \leq C\left(\left\|\partial_{t t} E\right\|_{L^{2}}^{2}+\left\|\partial_{x x} \partial_{t} E\right\|_{L^{2}}^{2}+\|n\|_{L^{\infty}}^{2}\left\|\partial_{t} E\right\|_{L^{2}}^{2}+\|E\|_{L^{\infty}}^{2}\left\|\partial_{t} n\right\|_{L^{2}}^{2}\right) \\
& \leq C\left(\left\|\partial_{t t} E\right\|_{L^{2}}^{2}+\left\|\partial_{x x} \partial_{t} E\right\|_{L^{2}}^{2}+1\right) .
\end{aligned}
$$

By using the $\epsilon$-Young inequality and the Gagliardo-Nirenberg inequality, we have

$$
\left\|\Lambda^{2 \alpha} \partial_{t} E\right\|_{L^{2}}^{2} \leq C\left\|\partial_{t t} E\right\|_{L^{2}}^{2}+\frac{1}{2}\left\|\Lambda^{2 \alpha} \partial_{t} E\right\|_{L^{2}}^{2}+C .
$$

Thus

$$
\left\|\Lambda^{2 \alpha} \partial_{t} E\right\|_{L^{2}}^{2} \leq C\left(\left\|\partial_{t t} E\right\|_{L^{2}}^{2}+1\right) .
$$

Combining (15) and (16) leads us to the estimate

$$
\begin{aligned}
& \frac{d}{d t}\left(\left\|\partial_{t t} n\right\|_{L^{2}}^{2}+\left\|\partial_{x} \partial_{t} n\right\|_{L^{2}}^{2}+H^{2}\left\|\Lambda^{\beta} \partial_{t} n\right\|_{L^{2}}^{2}\right) \\
& \quad \leq C\left(\left\|\partial_{t t} n\right\|_{L^{2}}^{2}+\left\|\partial_{t t} E\right\|_{L^{2}}^{2}+1\right) .
\end{aligned}
$$


Differentiating (5) with respect to $t$ twice, multiplying it by $\overline{\partial_{t t} E}$, integrating over $\Omega$, and then taking the imaginary part, we have

$$
\frac{1}{2} \frac{d}{d t}\left\|\partial_{t t} E\right\|_{L^{2}}^{2}-\operatorname{Im} \int_{\Omega} \partial_{t t}(n E) \overline{\partial_{t t} E} d x=0
$$

Therefore

$$
\begin{aligned}
\frac{d}{d t}\left\|\partial_{t t} E\right\|_{L^{2}}^{2} & \leq C\left(\left\|\partial_{t t} E\right\|_{L^{2}}^{2}+\left\|\partial_{t t} n\right\|_{L^{2}}^{2}+\left\|\partial_{t} E \partial_{t} n\right\|_{L^{2}}^{2}\right) \\
& \leq C\left(\left\|\partial_{t t} E\right\|_{L^{2}}^{2}+\left\|\partial_{t t} n\right\|_{L^{2}}^{2}+\left\|\partial_{t} E\right\|_{L^{2}}^{2}\left\|\partial_{t} n\right\|_{L^{\infty}}^{2}\right) \\
& \leq C\left(\left\|\partial_{t t} E\right\|_{L^{2}}^{2}+\left\|\partial_{t t} n\right\|_{L^{2}}^{2}+\left\|\partial_{x} \partial_{t} n\right\|_{L^{2}}^{2}\right) .
\end{aligned}
$$

From (17) and (18), we have

$$
\begin{aligned}
& \frac{d}{d t}\left(\left\|\partial_{t t} E\right\|_{L^{2}}^{2}+\left\|\partial_{t t} n\right\|_{L^{2}}^{2}+\left\|\partial_{x} \partial_{t} n\right\|_{L^{2}}^{2}+H^{2}\left\|\Lambda^{\beta} \partial_{t} n\right\|_{L^{2}}^{2}\right) \\
& \quad \leq C\left(\left\|\partial_{t t} E\right\|_{L^{2}}^{2}+\left\|\partial_{t t} n\right\|_{L^{2}}^{2}+\left\|\partial_{x} \partial_{t} n\right\|_{L^{2}}^{2}+1\right)
\end{aligned}
$$

Using the Gronwall inequality, we have

$$
\sup _{0 \leq t \leq T}\left(\left\|\partial_{t t} E\right\|_{L^{2}}^{2}+\left\|\partial_{t t} n\right\|_{L^{2}}^{2}+\left\|\Lambda^{\beta} \partial_{t} n\right\|_{L^{2}}^{2}\right) \leq C .
$$

Therefore, from (16), we can obtain

$$
\sup _{0 \leq t \leq T}\left\|\Lambda^{2 \alpha} \partial_{t} E\right\|_{L^{2}}^{2} \leq C .
$$

From (5)-(7), we can also get the following estimates easily:

$$
\sup _{0 \leq t \leq T}\left(\left\|\partial_{t t} \phi\right\|_{L^{2}}^{2}+\left\|\Lambda^{4 \alpha} E\right\|_{L^{2}}^{2}+\left\|\Lambda^{2 \beta} n\right\|_{L^{2}}^{2}+\left\|\Lambda^{\beta+2} \phi\right\|_{L^{2}}^{2}\right) \leq C .
$$

Thus we complete the proof.

Lemma 5 Assume that $E_{0}(x) \in H^{2 k \alpha}(\Omega), n_{0}(x) \in H^{k \beta}(\Omega), \phi_{0}(x) \in H^{2+(k-1) \beta}(\Omega), k \geq 2$. Then

$$
\sup _{0 \leq t \leq T}\left(\left\|\left(\partial_{t}\right)^{k} E\right\|_{L^{2}}^{2}+\left\|\left(\partial_{t}\right)^{k} n\right\|_{L^{2}}^{2}+\left\|\left(\partial_{t}\right)^{k} \phi\right\|_{L^{2}}^{2}\right) \leq C
$$

and

$$
\sup _{0 \leq t \leq T}\left(\left\|\Lambda^{2 \alpha}\left(\partial_{t}\right)^{k-1} E\right\|_{L^{2}}^{2}+\left\|\Lambda^{\beta}\left(\partial_{t}\right)^{k-1} n\right\|_{L^{2}}^{2}+\left\|\partial_{x x}\left(\partial_{t}\right)^{k-1} \phi\right\|_{L^{2}}^{2}\right) \leq C
$$

where $C$ is a positive constant depending on the initial data and $T$.

Proof The proof is by induction on $k$, the case $k=2$ being Lemma 4 above.

Assume now the lemma is valid for some integer $k \geq 2$, and suppose then

$$
E_{0}(x) \in H^{2 m \alpha}(\Omega), \quad n_{0}(x) \in H^{m \beta}(\Omega), \quad \phi_{0}(x) \in H^{2+(m-1) \beta}(\Omega), \quad m=k+1 .
$$


Differentiating (5) $(k+1)$-times with respect to $t$, then multiplying it by $\overline{\left(\partial_{t}\right)^{k+1} E}$, integrating over $\Omega$, and taking the imaginary part, we have

$$
\frac{1}{2} \frac{d}{d t}\left\|\left(\partial_{t}\right)^{k+1} E\right\|_{L^{2}}^{2}=\operatorname{Im} \int_{\Omega}\left(\partial_{t}\right)^{k+1}(n E) \overline{\left(\partial_{t}\right)^{k+1} E} d x
$$

Therefore

$$
\begin{aligned}
\frac{d}{d t}\left\|\left(\partial_{t}\right)^{k+1} E\right\|_{L^{2}}^{2} & =2 \operatorname{Im} \int_{\Omega}\left(\partial_{t}\right)^{k+1}(n E) \overline{\left(\partial_{t}\right)^{k+1} E} d x \\
& \leq C\left(\left\|\left(\partial_{t}\right)^{k+1} n\right\|_{L^{2}}^{2}+\left\|\left(\partial_{t}\right)^{k+1} E\right\|_{L^{2}}^{2}+1\right) .
\end{aligned}
$$

Differentiating (6) $(k+1)$-times with respect to $t$, then multiplying it by $\left(\partial_{t}\right)^{k+1} n$, and integrating over $\Omega$, we have

$$
\begin{aligned}
\frac{1}{2} & \frac{d}{d t}\left(\left\|\left(\partial_{t}\right)^{k+1} n\right\|_{L^{2}}^{2}+\left\|\partial_{x}\left(\partial_{t}\right)^{k} n\right\|_{L^{2}}^{2}+H^{2}\left\|\Lambda^{\beta}\left(\partial_{t}\right)^{k} n\right\|_{L^{2}}^{2}\right) \\
\quad & \int_{\Omega} \partial_{x x}\left(\partial_{t}\right)^{k}\left(|E|^{2}\right)\left(\partial_{t}\right)^{k+1} n d x
\end{aligned}
$$

Therefore

$$
\begin{aligned}
& \frac{d}{d t}\left(\left\|\left(\partial_{t}\right)^{k+1} n\right\|_{L^{2}}^{2}+\left\|\partial_{x}\left(\partial_{t}\right)^{k} n\right\|_{L^{2}}^{2}+H^{2}\left\|\Lambda^{\beta}\left(\partial_{t}\right)^{k} n\right\|_{L^{2}}^{2}\right) \\
& \quad \leq C\left(\left\|\left(\partial_{t}\right)^{k+1} n\right\|_{L^{2}}^{2}+\left\|\partial_{x x}\left(\partial_{t}\right)^{k}\left(|E|^{2}\right)\right\|_{L^{2}}^{2}\right) .
\end{aligned}
$$

\section{Since}

$$
i\left(\partial_{t}\right)^{k+1} E+\partial_{x x}\left(\partial_{t}\right)^{k} E-H^{2} \Lambda^{2 \alpha}\left(\partial_{t}\right)^{k} E=\left(\partial_{t}\right)^{k}(n E)
$$

it follows that

$$
\begin{aligned}
\left\|\Lambda^{2 \alpha}\left(\partial_{t}\right)^{k} E\right\|_{L^{2}}^{2} & \\
\leq & C\left(\left\|\left(\partial_{t}\right)^{k+1} E\right\|_{L^{2}}^{2}+\left\|\partial_{x x}\left(\partial_{t}\right)^{k} E\right\|_{L^{2}}^{2}+\left\|\left(\partial_{t}\right)^{k}(n E)\right\|_{L^{2}}^{2}\right) \\
\leq & C\left(\left\|\left(\partial_{t}\right)^{k+1} E\right\|_{L^{2}}^{2}+\left\|\left(\partial_{x x}\right)^{\alpha}\left(\partial_{t}\right)^{k} E\right\|_{L^{2}}^{\frac{2}{\alpha}}\left\|\left(\partial_{t}\right)^{k} E\right\|_{L^{2}}^{2-\frac{2}{\alpha}}\right. \\
& \left.+\left\|\left(\partial_{t}\right)^{k} n E\right\|_{L^{2}}^{2}+\sum_{i+j=k, 0<i<k, 0<j<k}\left\|\left(\partial_{t}\right)^{i} n\left(\partial_{t}\right)^{j} E\right\|_{L^{2}}^{2}+\left\|n\left(\partial_{t}\right)^{k} E\right\|_{L^{2}}^{2}\right) \\
\leq & C\left\|\left(\partial_{t}\right)^{k+1} E\right\|_{L^{2}}^{2}+\frac{1}{2}\left\|\left(\partial_{x x}\right)^{\alpha}\left(\partial_{t}\right)^{k} E\right\|_{L^{2}}^{2}+C+\left\|\left(\partial_{t}\right)^{k} n\right\|_{L^{2}}^{2}\|E\|_{L^{\infty}}^{2} \\
& +\sum_{i+j=k, 0<i<k, 0<j<k}\left\|\left(\partial_{t}\right)^{i} n\right\|_{L^{\infty}}^{2}\left\|\left(\partial_{t}\right)^{j} E\right\|_{L^{2}}^{2}+\|n\|_{L^{\infty}}^{2}\left\|\left(\partial_{t}\right)^{k} E\right\|_{L^{2}}^{2} \\
\leq & C\left\|\left(\partial_{t}\right)^{k+1} E\right\|_{L^{2}}^{2}+\frac{1}{2}\left\|\left(\partial_{x x}\right)^{\alpha}\left(\partial_{t}\right)^{k} E\right\|_{L^{2}}^{2}+\sum_{i+j=k, 0<i<k, 0<j<k}\left\|\Lambda^{\beta}\left(\partial_{t}\right)^{i} n\right\|_{L^{2}}^{2}\left\|\left(\partial_{t}\right)^{j} E\right\|_{L^{2}}^{2}+C \\
\leq & C\left\|\left(\partial_{t}\right)^{k+1} E\right\|_{L^{2}}^{2}+\frac{1}{2}\left\|\left(\partial_{x x}\right)^{\alpha}\left(\partial_{t}\right)^{k} E\right\|_{L^{2}}^{2}+C,
\end{aligned}
$$


i.e.

$$
\left\|\Lambda^{2 \alpha}\left(\partial_{t}\right)^{k} E\right\|_{L^{2}}^{2} \leq C\left\|\left(\partial_{t}\right)^{k+1} E\right\|_{L^{2}}^{2}+C
$$

Therefore

$$
\begin{aligned}
\left\|\partial_{x x}\left(\partial_{t}\right)^{k} E\right\|_{L^{2}}^{2} & \leq C\left\|\Lambda^{2 \alpha}\left(\partial_{t}\right)^{k} E\right\|_{L^{2}}^{\frac{2}{\alpha}}\left\|\left(\partial_{t}\right)^{k} E\right\|_{L^{2}}^{2-\frac{2}{\alpha}} \leq C\left(\left\|\Lambda^{2 \alpha}\left(\partial_{t}\right)^{k} E\right\|_{L^{2}}^{2}+1\right) \\
& \leq C\left(\left\|\left(\partial_{t}\right)^{k+1} E\right\|_{L^{2}}^{2}+1\right) .
\end{aligned}
$$

Substituting it into (20), we have

$$
\begin{aligned}
& \frac{d}{d t}\left(\left\|\left(\partial_{t}\right)^{k+1} n\right\|_{L^{2}}^{2}+\left\|\partial_{x}\left(\partial_{t}\right)^{k} n\right\|_{L^{2}}^{2}+H^{2}\left\|\Lambda^{\beta}\left(\partial_{t}\right)^{k} n\right\|_{L^{2}}^{2}\right) \\
& \quad \leq C\left(\left\|\left(\partial_{t}\right)^{k+1} n\right\|_{L^{2}}^{2}+\left\|\left(\partial_{t}\right)^{k+1} E\right\|_{L^{2}}^{2}+1\right) .
\end{aligned}
$$

From (19) and (21), we have

$$
\begin{aligned}
& \frac{d}{d t}\left(\left\|\left(\partial_{t}\right)^{k+1} E\right\|_{L^{2}}^{2}+\left\|\left(\partial_{t}\right)^{k+1} n\right\|_{L^{2}}^{2}+\left\|\partial_{x}\left(\partial_{t}\right)^{k} n\right\|_{L^{2}}^{2}+H^{2}\left\|\Lambda^{\beta}\left(\partial_{t}\right)^{k} n\right\|_{L^{2}}^{2}\right) \\
& \quad \leq C\left(\left\|\left(\partial_{t}\right)^{k+1} n\right\|_{L^{2}}^{2}+\left\|\left(\partial_{t}\right)^{k+1} E\right\|_{L^{2}}^{2}+1\right) .
\end{aligned}
$$

Thus the Gronwall inequality yields

$$
\left\|\left(\partial_{t}\right)^{k+1} E\right\|_{L^{2}}^{2}+\left\|\left(\partial_{t}\right)^{k+1} n\right\|_{L^{2}}^{2}+\left\|\partial_{x}\left(\partial_{t}\right)^{k} n\right\|_{L^{2}}^{2}+H^{2}\left\|\Lambda^{\beta}\left(\partial_{t}\right)^{k} n\right\|_{L^{2}}^{2} \leq C .
$$

Then from (7), we can deduce that

$$
\left\|\partial_{x x}\left(\partial_{t}\right)^{k} \phi\right\|_{L^{2}}^{2} \leq C .
$$

Since

$$
\left(\partial_{t}\right)^{k+1} \phi-\left(\partial_{t}\right)^{k} n-H^{2} \Lambda^{2(\beta-1)}\left(\partial_{t}\right)^{k} n-\left(\partial_{t}\right)^{k}\left(|E|^{2}\right)=0,
$$

we have

$$
\left\|\left(\partial_{t}\right)^{k+1} \phi\right\|_{L^{2}}^{2} \leq C\left(\left\|\left(\partial_{t}\right)^{k} n\right\|_{L^{2}}^{2}+\left\|\Lambda^{2(\beta-1)}\left(\partial_{t}\right)^{k} n\right\|_{L^{2}}^{2}+\left\|\left(\partial_{t}\right)^{k}\left(|E|^{2}\right)\right\|_{L^{2}}^{2}\right) \leq C,
$$

where we have used the fact $\beta-1<\frac{\beta}{2}$. The proof is complete.

\section{The existence result of the solution}

Now we state our main result as follows.

Theorem 1 Assume that $1<\alpha \leq \beta<2$, and $E_{0}(x) \in H^{2 \alpha}(\Omega), n_{0}(x) \in H^{\beta}(\Omega), \phi_{0}(x) \in$ $H^{2}(\Omega)$. Then there exists a unique global solution to problem (5)-(9),

$$
\begin{aligned}
& E \in L^{\infty}\left(0, T ; H^{2 \alpha}\right), \quad n \in L^{\infty}\left(0, T ; H^{\beta}\right), \quad \phi \in L^{\infty}\left(0, T ; H^{2}\right), \\
& E_{t} \in L^{\infty}\left(0, T ; L^{2}\right), \quad n_{t} \in L^{\infty}\left(0, T ; L^{2}\right), \quad \phi_{t} \in L^{\infty}\left(0, T ; L^{2}\right) .
\end{aligned}
$$


Proof By using the Galerkin method, we find the approximate solution as follows

$$
E_{m}(t)=\sum_{j=0}^{m} g_{j m}(t) \omega_{j}, \quad n_{m}(t)=\sum_{j=0}^{m} h_{j m}(t) \omega_{j}, \quad \phi_{m}(t)=\sum_{j=0}^{m} l_{j m}(t) \omega_{j},
$$

where $\omega_{j}=e^{i j x}, 0 \leq j \leq m$. According to the Galerkin method, these undetermined coefficients $g_{j m}(t), h_{j m}(t), l_{j m}(t)$ need to satisfy the following initial boundary value problem of the system of ordinary differential equations

$$
\begin{aligned}
& \left(i \partial_{t} E_{m}+\partial_{x x} E_{m}-H^{2} \Lambda^{2 \alpha} E_{m}-n_{m} E_{m}, \omega_{j}\right)=0, \\
& \left(\partial_{t} n_{m}-\partial_{x x} \phi_{m}, \omega_{j}\right)=0, \\
& \left(\partial_{t} \phi_{m}-n_{m}+H^{2}\left(-\partial_{x x}\right)^{\beta-1} n_{m}-\left|E_{m}\right|^{2}, \omega_{j}\right)=0, \\
& E_{m}(x+2 \pi, t)=E_{m}(x, t), \quad n_{m}(x+2 \pi, t)=n_{m}(x, t), \\
& \phi_{m}(x+2 \pi, t)=\phi_{m}(x, t), \\
& E_{m}(x, 0)=E_{0 m}(x) \in \operatorname{span}\left\{\omega_{j}, 0 \leq j \leq m\right\}, \\
& n_{m}(x, 0)=n_{0 m}(x) \in \operatorname{span}\left\{\omega_{j}, 0 \leq j \leq m\right\}, \\
& \phi_{m}(x, 0)=\phi_{0 m}(x) \in \operatorname{span}\left\{\omega_{j}, 0 \leq j \leq m\right\},
\end{aligned}
$$

where $E_{0 m}(x) \stackrel{H^{2 \alpha}}{\longrightarrow} E_{0}(x), n_{0 m}(x) \stackrel{H^{\beta}}{\longrightarrow} n_{0}(x)$, and $\phi_{0 m}(x) \stackrel{H^{2}}{\longrightarrow} \phi_{0}(x)$ as $m \rightarrow \infty$. Similar to the proof of Lemma 3, we can deduce that the sequence $\left\{E_{m}\right\}_{m=1}^{\infty}$ is bounded in $L^{\infty}\left(0, T ; H^{2 \alpha}(\Omega)\right),\left\{n_{m}\right\}_{m=1}^{\infty}$ is bounded in $L^{\infty}\left(0, T ; H^{\beta}(\Omega)\right),\left\{\phi_{m}\right\}_{m=1}^{\infty}$ is bounded in $L^{\infty}\left(0, T ; H^{2}(\Omega)\right),\left\{E_{m t}\right\}_{m=1}^{\infty}$ is bounded in $L^{\infty}\left(0, T ; L^{2}(\Omega)\right),\left\{n_{m t}\right\}_{m=1}^{\infty}$ is bounded in $L^{\infty}(0, T$; $\left.L^{2}(\Omega)\right),\left\{\phi_{m t}\right\}_{m=1}^{\infty}$ is bounded in $L^{\infty}\left(0, T ; L^{2}(\Omega)\right)$.

By a compactness argument, we can choose subsequences, still denoted by $E_{m}(x, t)$, $n_{m}(x, t), \phi_{m}(x, t)$, such that

$$
\begin{aligned}
& E_{m}(x, t) \rightarrow E(x, t) \quad \text { in } L^{\infty}\left(0, T ; H^{2 \alpha}(\Omega)\right) \text { star weakly, } \\
& n_{m}(x, t) \rightarrow n(x, t) \quad \text { in } L^{\infty}\left(0, T ; H^{\beta}(\Omega)\right) \text { star weakly, } \\
& \phi_{m}(x, t) \rightarrow \phi(x, t) \quad \text { in } L^{\infty}\left(0, T ; H^{2}(\Omega)\right) \text { star weakly, } \\
& E_{m t}(x, t) \rightarrow E_{t}(x, t) \quad \text { in } L^{\infty}\left(0, T ; L^{2}(\Omega)\right) \text { star weakly, } \\
& n_{m t}(x, t) \rightarrow n_{t}(x, t) \quad \text { in } L^{\infty}\left(0, T ; L^{2}(\Omega)\right) \text { star weakly, } \\
& \phi_{m t}(x, t) \rightarrow \phi_{t}(x, t) \quad \text { in } L^{\infty}\left(0, T ; L^{2}(\Omega)\right) \text { star weakly, } \\
& E_{m}(x, t) \rightarrow E(x, t) \quad \text { in } L^{2}\left(0, T ; L^{2}(\Omega)\right) \text { strongly and a.e., } \\
& n_{m}(x, t) \rightarrow n(x, t) \quad \text { in } L^{2}\left(0, T ; L^{2}(\Omega)\right) \text { strongly and a.e., } \\
& n_{m}(x, t) E_{m}(x, t) \rightarrow n(x, t) E(x, t) \quad \text { in } L^{\infty}\left(0, T ; L^{2}(\Omega)\right) \text { star weakly, } \\
& \left|E_{m}(x, t)\right|^{2} \rightarrow|E(x, t)|^{2} \quad \text { in } L^{\infty}\left(0, T ; L^{2}(\Omega)\right) \text { star weakly. }
\end{aligned}
$$

By using the density of $\omega_{j}$ in $L^{2}$, we obtain the existence of a local solution for the problem (5)-(9). By the continuous extension principle, from the conditions of the theorem and 
a priori estimates in Section 2, we can get the existence of the global solution for the problem (5)-(9).

Next, we will show the uniqueness of the solution.

Suppose that there are two solutions $\left(E_{1}, n_{1}, \phi_{1}\right)$ and $\left(E_{2}, n_{2}, \phi_{2}\right)$. Let

$$
\widetilde{E}=E_{1}-E_{2}, \quad \widetilde{n}=n_{1}-n_{2}, \quad \widetilde{\phi}=\phi_{1}-\phi_{2},
$$

then we have

$$
\begin{aligned}
& i \partial_{t} \widetilde{E}+\partial_{x x} \widetilde{E}-H^{2} \Lambda^{2 \alpha} \widetilde{E}=n_{1} \widetilde{E}+\widetilde{n} E_{2}, \\
& \partial_{t} \widetilde{n}-\partial_{x x} \widetilde{\phi}=0, \\
& \partial_{t} \widetilde{\phi}-\widetilde{n}+H^{2} \Lambda^{2 \beta-2} \widetilde{n}=\left|E_{1}\right|^{2}-\left|E_{2}\right|^{2},
\end{aligned}
$$

with initial data

$$
\left.\widetilde{E}\right|_{t=0}=0,\left.\quad \widetilde{n}\right|_{t=0}=0,\left.\quad \widetilde{\phi}\right|_{t=0}=0,
$$

and periodic boundary conditions

$$
\widetilde{E}(x+2 \pi, t)=\widetilde{E}(x, t), \quad \widetilde{n}(x+2 \pi, t)=\widetilde{n}(x, t), \quad \widetilde{\phi}(x+2 \pi, t)=\widetilde{\phi}(x, t) .
$$

Multiplying (29) by $\widetilde{E}$, integrating over $\Omega$, and then taking the imaginary part, we have

$$
\begin{aligned}
\frac{d}{d t}\|\widetilde{E}\|_{L^{2}}^{2} & =2 \operatorname{Im} \int_{\Omega} \widetilde{n} E_{2} \widetilde{\widetilde{E}} d x \\
& \leq C\left\|E_{2}\right\|_{L^{\infty}}\|\widetilde{n}\|_{L^{2}}\|\widetilde{E}\|_{L^{2}} \\
& \leq C\left(\|\widetilde{n}\|_{L^{2}}^{2}+\|\widetilde{E}\|_{L^{2}}^{2}\right) .
\end{aligned}
$$

Differentiating (30) with respect to $t$, then multiplying it by $\partial_{t} \widetilde{n}$, integrating over $\Omega$, we have

$$
\frac{1}{2} \frac{d}{d t}\left(\left\|\partial_{t} \widetilde{n}\right\|_{L^{2}}^{2}+\left\|\partial_{x} \widetilde{n}\right\|_{L^{2}}^{2}+H^{2}\left\|\Lambda^{\beta} \widetilde{n}\right\|_{L^{2}}^{2}\right)=\int_{\Omega}\left(\partial_{x x}\right)\left(E_{1} \widetilde{\widetilde{E}}+\widetilde{E} \overline{E_{2}}\right) \partial_{t} \widetilde{n} d x
$$

Therefore

$$
\begin{aligned}
& \frac{d}{d t}\left(\left\|\partial_{t} \widetilde{n}\right\|_{L^{2}}^{2}+\left\|\partial_{x} \widetilde{n}\right\|_{L^{2}}^{2}+H^{2}\left\|\Lambda^{\beta} \widetilde{n}\right\|_{L^{2}}^{2}\right) \\
& \quad \leq C\left(\|\widetilde{E}\|_{L^{2}}+\left\|\partial_{x x} \widetilde{E}\right\|_{L^{2}}\right)\left\|\partial_{t} \widetilde{n}\right\|_{L^{2}} \\
& \quad \leq C\left(\|\widetilde{E}\|_{L^{2}}^{2}+\left\|\partial_{x x} \widetilde{E}\right\|_{L^{2}}^{2}+\left\|\partial_{t} \widetilde{n}\right\|_{L^{2}}^{2}\right) .
\end{aligned}
$$

Since

$$
\begin{aligned}
\left\|\Lambda^{2 \alpha} \widetilde{E}\right\|_{L^{2}} & \leq\left\|\partial_{t} \widetilde{E}\right\|_{L^{2}}+\left\|\partial_{x x} \widetilde{E}\right\|_{L^{2}}+\|\widetilde{n}\|_{L^{2}}\left\|E_{2}\right\|_{L^{\infty}}+\left\|n_{1}\right\|_{L^{\infty}}\|\widetilde{E}\|_{L^{2}} \\
& \leq\left\|\partial_{t} \widetilde{E}\right\|_{L^{2}}+\frac{1}{2}\left\|\Lambda^{2 \alpha} \widetilde{E}\right\|_{L^{2}}+C\|\widetilde{n}\|_{L^{2}}+C\|\widetilde{E}\|_{L^{2}}
\end{aligned}
$$


we have

$$
\left\|\Lambda^{2 \alpha} \widetilde{E}\right\|_{L^{2}} \leq C\left(\left\|\partial_{t} \widetilde{E}\right\|_{L^{2}}+\|\widetilde{E}\|_{L^{2}}+\|\widetilde{n}\|_{L^{2}}\right)
$$

Furthermore,

$$
\begin{aligned}
\left\|\partial_{x x} \widetilde{E}\right\|_{L^{2}}^{2} & \leq C\left(\left\|\Lambda^{2 \alpha} \widetilde{E}\right\|_{L^{2}}^{2}+\|\widetilde{E}\|_{L^{2}}^{2}\right) \\
& \leq C\left(\left\|\partial_{t} \widetilde{E}\right\|_{L^{2}}^{2}+\|\widetilde{E}\|_{L^{2}}^{2}+\|\widetilde{n}\|_{L^{2}}^{2}\right) .
\end{aligned}
$$

Substituting it into (33), we have

$$
\begin{aligned}
& \frac{1}{2} \frac{d}{d t}\left(\left\|\partial_{t} \widetilde{n}\right\|_{L^{2}}^{2}+\left\|\partial_{x} \widetilde{n}\right\|_{L^{2}}^{2}+H^{2}\left\|\Lambda^{\beta} \widetilde{n}\right\|_{L^{2}}^{2}\right) \\
& \quad \leq C\left(\left\|\partial_{t} \widetilde{E}\right\|_{L^{2}}^{2}+\|\widetilde{E}\|_{L^{2}}^{2}+\|\widetilde{n}\|_{L^{2}}^{2}+\left\|\partial_{t} \widetilde{n}\right\|_{L^{2}}^{2}\right) .
\end{aligned}
$$

Differentiating (29) with respect to $t$, we have

$$
i \partial_{t t} \widetilde{E}+\partial_{x x} \partial_{t} \widetilde{E}-H^{2} \Lambda^{2 \alpha} \partial_{t} \widetilde{E}=\partial_{t} n_{1} \widetilde{E}+n_{1} \partial_{t} \widetilde{E}+\partial_{t} \widetilde{n} E_{2}+\widetilde{n} \partial_{t} E_{2} .
$$

Multiplying (35) by $\partial_{t} \widetilde{\widetilde{E}}$, integrating over $\Omega$, and taking the imaginary part, we have

$$
\begin{aligned}
\frac{1}{2} \frac{d}{d t}\left\|\partial_{t} \widetilde{E}\right\|_{L^{2}}^{2} \leq & \left\|\partial_{t} n_{1}\right\|_{L^{\infty}}\|\widetilde{E}\|_{L^{2}}\left\|\partial_{t} \widetilde{E}\right\|_{L^{2}} \\
& +\left\|\partial_{t} \widetilde{n}\right\|_{L^{2}}\left\|E_{2}\right\|_{L^{\infty}}\left\|\partial_{t} \widetilde{E}\right\|_{L^{2}}+\|\widetilde{n}\|_{L^{2}}\left\|\partial_{t} E_{2}\right\|_{L^{\infty}}\|\| \partial_{t} \widetilde{E} \|_{L^{2}} \\
\leq & C\left(\|\widetilde{E}\|_{L^{2}}^{2}+\left\|\partial_{t} \widetilde{E}\right\|_{L^{2}}^{2}+\|\widetilde{n}\|_{L^{2}}^{2}+\left\|\partial_{t} \widetilde{n}\right\|_{L^{2}}^{2}\right) .
\end{aligned}
$$

Recall the inequality

$$
\frac{1}{2} \frac{d}{d t}\|\widetilde{n}\|_{L^{2}}^{2} \leq\|\widetilde{n}\|_{L^{2}}\left\|\partial_{t} \widetilde{n}\right\|_{L^{2}} \leq C\left(\|\widetilde{n}\|_{L^{2}}^{2}+\left\|\partial_{t} \widetilde{n}\right\|_{L^{2}}^{2}\right) .
$$

Combining (32), (34), (36) and (37), we have

$$
\begin{aligned}
& \frac{d}{d t}\left(\|\widetilde{E}\|_{L^{2}}^{2}+\left\|\partial_{t} \widetilde{E}\right\|_{L^{2}}^{2}+\|\widetilde{n}\|_{L^{2}}^{2}+\left\|\partial_{t} \widetilde{n}\right\|_{L^{2}}^{2}+\left\|\partial_{x} \widetilde{n}\right\|_{L^{2}}^{2}+H^{2}\left\|\Lambda^{\beta} \widetilde{n}\right\|_{L^{2}}^{2}\right) \\
& \quad \leq C\left(\|\widetilde{E}\|_{L^{2}}^{2}+\left\|\partial_{t} \widetilde{E}\right\|_{L^{2}}^{2}+\|\widetilde{n}\|_{L^{2}}^{2}+\left\|\partial_{t} \widetilde{n}\right\|_{L^{2}}^{2}+\left\|\partial_{x} \widetilde{n}\right\|_{L^{2}}^{2}+H^{2}\left\|\Lambda^{\beta} \widetilde{n}\right\|_{L^{2}}^{2}\right) .
\end{aligned}
$$

Using the Gronwall inequality and initial data, we deduce

$$
\widetilde{n}=0, \quad \widetilde{E}=0, \quad \widetilde{\phi}=0 .
$$

Theorem 2 Assume that $1<\alpha \leq \beta<2$, and $E_{0}(x) \in H^{2 k \alpha}(\Omega), n_{0}(x) \in H^{k \beta}(\Omega), \phi_{0}(x) \in$ $H^{2+(k-1) \beta}(\Omega), k \geq 2$. Then there exists a unique global solution to (5)-(9).

Proof Using Lemma 5 and the embedding theory of Sobolev spaces, we can prove the theorem above. Since the proof is similar to the proof of Theorem 1, we omit it. 


\section{Conclusions}

In the past decades, quantum plasmas have been attracting considerable attention, both from the physical and mathematical viewpoints. In this paper, we have considered the global existence of the smooth solutions to the fractional modified Zakharov system, when the quantum correction was taken into account. For the other mathematical property of the fractional modified Zakharov system, it is a subject which is still very open to new developments. We will consider the numerical solutions to the fractional modified Zakharov system in a coming study.

\section{Competing interests}

The authors declare that they have no competing interests.

\section{Authors' contributions}

The authors contributed equally to the writing of this paper. All authors read and approved the final manuscript.

\section{Acknowledgements}

Lang Li was partially supported by the NSFC under grant No. 11426069, No. 11401223 and No. 61375006. Lingyu Jin was supported by the NSFC under grant No. 11101160. Shaomei Fang was supported by the NSFC under grant No. 11271141.

Received: 21 September 2015 Accepted: 1 December 2015 Published online: 15 December 2015

\section{References}

1. Zakharov, VE: The collapse of Langmuir waves. Sov. Phys. JETP 35, 908-914 (1972)

2. de Oliveira, Gl, Rizzato, FB: Phase-locking and resonant islands in the Zakharov equations. Phys. Lett. A 214, 40-46 (1996)

3. Fedele, R, Shukla, PK, Onoratoc, M, Anderson, D, Lisak, M: Landau damping of partially incoherent Langmuir waves. Phys. Lett. A 303, 61-66 (2002)

4. Misra, AP, Ghoshb, D, Chowdhury, AR: A novel hyperchaos in the quantum Zakharov system for plasmas. Phys. Lett. A 372, 1469-1476 (2008)

5. Bourgain, J, Colliander, J: On well-posedness of Zakharov system. Int. Math. Res. Not. 11, 515-546 (1996)

6. Ginibre, J, Tsutsumi, Y, Velo, G: On the Cauchy problem for Zakharov system. J. Funct. Anal. 151, $384-436$ (1997)

7. Bejenaru, I, Herr, S, Holmer, J, Tataru, D: On the 2 d Zakharov system with L² Schrödinger data. Nonlinearity 22 1063-1089 (2009)

8. Pecher, $\mathrm{H}$ : Global solutions with infinite energy for the one-dimensional Zakharov system. Electron. J. Differ. Equ. 2005, 41 (2005)

9. Garcia, LG, Haas, F, de Oliveira, LPL, Goedert, J: Modified Zakharov equations for plasmas with a quantum correction. Phys. Plasmas 12, 012302 (2005)

10. Markowich, PA, Ringhofer, CA, Schmeiser, C: Semiconductor Equations. Springer, Vienna (2002)

11. Guo, CH, Fang, SM, Guo, BL: Long time behavior of the solutions for the dissipative modified Zakharov equations for plasmas with a quantum correction. J. Math. Anal. Appl. 403, 183-192 (2013)

12. Wang, G, Ahmad, B, Zhang, L: Impulsive anti-periodic boundary value problem for nonlinear differential equations of fractional order. Nonlinear Anal. 74, 792-804 (2011)

13. Samko, SG, Kilbas, AA, Marichev, Ol: Fractional Integrals and Derivatives, Theory \& Applications. Gordon and Breach, Yverdon (1993)

14. Podlubny, I: Fractional Differential Equations. Academic Press, San Diego (1999)

15. Kilbas, AA, Srivastava, HM, Trujillo, JJ: Theory and Applications of Fractional Differential Equations. North-Holland Mathematics Studies, vol. 204. Elsevier, Amsterdam (2006)

16. Golmankhaneh, AK, Arefi, R, Baleanu, D: The proposed modified Liu system with fractional order. Adv. Math. Phys. 2013, 186037 (2013)

17. Zhou, Y, Jiao, F, Li, J: Existence and uniqueness for fractional neutral differential equations with infinite delay. Nonlinear Anal. 71, 3249-3256 (2009)

18. Zhou, Y, Jiao, F, Li, J: Existence and uniqueness for p-type fractional neutral differential equations. Nonlinear Anal. 71, 2724-2733 (2009)

19. Wang, J, Zhou, Y: A class of fractional evolution equations and optimal controls. Nonlinear Anal., Real World Appl. 12 , 262-272 (2011)

20. Tian, YS, Bai, ZB: Existence results for the three-point impulsive boundary value problem involving fractional differential equations. Comput. Math. Appl. 59, 2601-2609 (2010)

21. Baleanu, D, Diethelm, K, Scalas, E, Trujillo, JJ: Models and Numerical Methods, vol. 3. World Scientific, Singapore (2012)

22. Liu, Y: Existence and uniqueness of solutions for a class of initial value problems of fractional differential systems on half lines. Bull. Sci. Math. 137, 1048-1071 (2013)

23. Kosmatov, N: Integral equations and initial value problems for nonlinear differential equations of fractional order. Nonlinear Anal. 70, 2521-2529 (2009)

24. Guo, BL, Han, YQ, Xin, J: Existence of the global smooth solution to the period boundary value problem of fractional nonlinear Schrödinger equation. Appl. Math. Comput. 204, 468-477 (2008)

25. You, SJ, Guo, BL, Ning, XQ: Initial boundary value problem for modified Zakharov equations. Acta Math. Sci. 32 , 1455-1466 (2012) 
26. Fang, SM, Jin, LY, Guo, BL: Existence of weak solution for quantum Zakharov equations for plasmas mode. Appl. Math. Mech. 32(10), 1339-1344 (2011)

27. Jin, LY, Fang, SM, Guo, BL: Existence of attractors for modified Zakharov equations for plasmas with a quantum correction. J. Math. Phys. 53, 072703 (2012)

Submit your manuscript to a SpringerOpen ${ }^{\odot}$ journal and benefit from:

- Convenient online submission

Rigorous peer review

- Immediate publication on acceptance

- Open access: articles freely available online

- High visibility within the field

- Retaining the copyright to your article

Submit your next manuscript at springeropen.com 